\section{High fructose intake fails to induce symptomatic adaptation but may induce intestinal carriers}

\author{
Debra Heilpern, ${ }^{1}$ Rateb N. Abbas ${ }^{2}$ \\ Stephanie Gladman, ${ }^{3}$ Maryse Menard, ${ }^{3}$ \\ Byong H. Lee, ${ }^{4}$ Andrew Szilagyi' \\ 'Jewish General Hospital, Division of \\ Gastroenterology, Mcgill University School \\ of Medicine, Montreal, QC, Canada \\ 2Department of Microbial Biotechnology, \\ Genetic Engineering and Biotechnology \\ Research Institute (GEBRI), Minufiya \\ University, Egypt \\ ${ }^{3}$ Jewish General Hospital, Department of \\ Dietetics, Mcgill University, Montreal, QC, \\ Canada \\ ${ }^{4}$ Department of Microbiology and \\ Immunology, McGill University, Montreal, \\ QC, Canada
}

\section{Abstract}

Fructose has several interactions in man, including intolerance and promotion of some diseases. However, fructose in fruits and in prebiotics may be associated with benefits. Adaptation to regular fructose ingestion as defined for lactose could support a beneficial rather than a deleterious effect. This study was undertaken to evaluate symptomatic response and potential underlying mechanisms of fecal bacterial change and breath hydrogen response to short term regular fructose supplementation.

Forty-five participants were recruited for a 3 day recall diet questionnaire and a $50 \mathrm{~g}$ fructose challenge. Breath hydrogen was measured for $4.5 \mathrm{hrs}$ and symptoms were recorded. Thirtyeight subjects provided stool samples for analysis by selective culture of 4 groups of bacteria, including bifidobacteria and lactobacilli. Intolerant subjects returned a second time 15 days later. Ten of these served as controls and 16 received $30 \mathrm{~g}$ fructose twice a day. Ten of the latter returned 27 days later, after stopping fructose for a third challenge test. Student's paired, unpaired t-tests and Pearson correlations were used. Significance was accepted at $\mathrm{P}<0.05$.

After fructose rechallenge there were no significant reductions in symptoms scores in volunteers in either the fructose supplemented or non supplemented groups. However, total breath hydrogen was reduced between test 1 and test $2(\mathrm{P}=0.03)$ or test $3(\mathrm{P}=0.04)$ in the group given fructose then discontinued, compared with controls. There were no statistically significant changes in bacterial numbers between test 2 and 1 .

This study shows that regular consumption of high dose fructose does not follow the lactose model of adaptation. Observed changes in hydrogen breath tests raise the possibility that intestinal carriers of fructose may be induced potentially aggravating medical problems attributed to fructose.

\section{Introduction}

Fructose, glucose and galactose represent the main monosaccharides utilized by man and most mammals. However, there is a dose dependent intolerance to consumption of fructose and this may contribute to symptoms of irritable bowel syndrome..$^{1-3}$ Furthermore, fructose is also putatively blamed for the current epidemic of obesity, metabolic syndrome and a number of other diseases. ${ }^{47}$ Nevertheless, polymers of fructose constitute some of the most important prebiotics supporting the growth of health promoting bacteria. ${ }^{8-10}$ In addition, fructose is the main sugar in fruits, which along with vegetables, may also protect against some cancers. $^{11,12}$

Fructose intolerance results from malabsorbed sugar which reaches the colon and is metabolized by bacteria. Rapid entry and possible osmotic forces created by the process lead to symptoms of gas, bloating, cramps and at times diarrhea. These are similar to symptoms induced by malabsorbed sugars like lactose or lactulose. It has been reported with these that continued regular consumption leads to adaptation. ${ }^{13,14}$ The consequence is improved symptoms of tolerance. Because lactase is a non inducible enzyme, ${ }^{15}$ the process of improvement depends on altered microbial metabolism. These could be clinically measured by evaluating quantitative microbial changes and followed physiologically by alterations in breath hydrogen. ${ }^{14}$ Together with such a process, it could be construed as a prebiotic effect. ${ }^{16}$ This triple combination for lactose (and lactulose) constitutes a definition of adaptation with potential benefits.

If bacterial changes similar to other prebiotics could be assigned to fructose these effects would need to be considered in the prevention of some diseases. It is not clear however, whether adaptation to continued regular fructose could lead to changes similar to lactose (as described above). ${ }^{17}$ In an earlier report examining adaptation parameters for fructose, none could be found.$^{18}$ However, the study evaluated patients consuming generally less than $25 \mathrm{~g}$ daily. Subsequently, it was reported that colonic spillage, a necessary prerequisite to adaptation, occurs after a $25 \mathrm{~g}$ dose ingesting in one meal. ${ }^{19}$

This study was therefore undertaken to determine if adaptation features could be induced by daily high dose fructose in intolerant healthy volunteers. The first goal was to determine whether symptoms could be reduced as
Correspondence: Dr A. Szilagyi, Jewish General Hospital, 3755 Cote St Catherine Rd, Room G327 Montreal H3T 1E2, QC, Canada

Tel: +1.514.340 8144. Fax: +1.514.340 8282

E-mail: aszilagy@gas.jgh.mcgill.ca

Key words: fructose tolerance, colonic microflora, intestinal carriers.

Funding: financial assistance was provided by departmental funds.

Acknowledgement: Professor Brian Smith Desautels Faculty of Management, McGill university for reviewing statistics of the manuscript.

Conflicts of interest: the author reports no conflicts of interest.

Contributions: DH was responsible for the conduct of the study, planning, statistical analysis and participated in writing the manuscript; RA and BL were responsible for carrying out and analysing all microbiological data; SG and MM were responsible for recording and calculating diet pertaining data; AS was responsible for conceiving the project, helping analyze the data and writing the paper. All authors participated in discussions and approve the final submitted manuscript.

Received for publication: 22 October 2009. Revision received: 13 December 2009.

Accepted for publication: 13 December 2009.

This work is licensed under a Creative Commons Attribution 3.0 License (by-nc 3.0).

(C) Copyright D. Heilpern et al., 2010

Licensee PAGEPress, Italy

Gastroenterology Insights 2010; 2:e3

doi:10.4081/gi.2010.e3

with lactose, because this aspect is the most clinically relevant. Secondary goals were to evaluate mechanisms whereby adaptation could occur. In view of the importance of fructose polymers in prebiotic production, we were particularly interested whether monosaccharide fructose could have any effects on bifidobacteria and lactobacilli. To this end the hydrogen breath test is a physiological monitor of events involved with sugar handling after ingestion. Levels may be affected by reduced bacterial production or increased intestinal absorption of the sugar. Symptoms did not improve after repeat fructose challenges. Similarly no significant bacterial changes occurred. However, unexpectedly there was a significant reduction in breath hydrogen on rechallenges.

\section{Materials and Methods}

Healthy men and women aged 18-50 years were recruited through advertising within the 
institution and at a university. Antibiotics at least 1 month prior or during the study were prohibited. Pregnancy or any disorder except thyroid hormone replacement and cholesterol reducing agents resulted in exclusion. Volunteers were informed of the study and signed written consent after approval by the Ethics Research Board of the Jewish General Hospital and was registered with clinicaltrials.gov, identifier no. NCT00775567.

\section{Outline of study}

The rationale of tests evaluated in this study are based on the definition of colonic adaptation as described by Hertzler and Savaiano. ${ }^{13}$ Following regular lactose consumption a second challenge test is performed and both a reduction of symptom score and sum of total breath hydrogen (over the test) is observed. In addition, bacterial effect was originally evaluated by measuring fecal $\beta$-galactosidase. Here we substitute actual bacterial species, looking for a rise in bifidobacteria and lactobacilli on the second challenge.

The reason for the diet questionnaire is to evaluate whether under real life conditions daily fructose intake influences results of fructose challenge tests, as seen for lactose intolerance. ${ }^{18}$

After a low carbohydrate supper and overnight fast (except water), the volunteers filled in a previously validated 3 day diet questionnaire targeting daily fructose containing nutrients..$^{20}$ Smoking at any time was prohibited and $50 \mathrm{~g}$ fructose dissolved in $250 \mathrm{~mL}$ of water (25\% solution) was consumed over several minutes. Breath hydrogen and symptoms were recorded each $30 \mathrm{~min}$ for $4.5 \mathrm{hrs}$. Stool samples were obtained during the test and sent for bacterial analysis to the Macdonald campus laboratory.

Intolerant volunteers were asked to return for a second similar set (except diet questionnaire). In a random but unblinded order based on alternate day results of tests of volunteers, part received $30 \mathrm{~g}$ BID of fructose for 2 weeks (Group F) and others received no additional fructose (Group Never F) and returned also at a median of 15 days. Additional containers were given if

Table 1. Demographic features of fructose tolerant and intolerant volunteers.

\begin{tabular}{lcc} 
& Tolerant & Intolerant \\
Age (mean \pm SD years) & $30.1 \pm 9.1$ & $29.2 \pm 8.3$ \\
Male & 6 & 14 \\
\hline Female & 9 & 16 \\
White & 10 & 13 \\
\hline Black & 1 & 2 \\
Asian & 4 & 5 \\
\hline Mean \pm SD fructose $(\mathrm{g} / \mathrm{d})$ & $12.9 \pm 8.9$ & $13.4 \pm 8.9$ \\
\hline Demographic features of fructose tolerant (N15) and intolerant (N30) participants are shown. None of the differences were statistically sig- &
\end{tabular}

they could not return on the date selected. Compliance was inadequately monitored, unfortunately, with only about $50 \%$ returning empty containers. However, historically all claimed having ingested the fructose given. Some Group $\mathrm{F}$ participants returned for a third set of tests after a median of 27 days having discontinued fructose (Group Stop F). Neither diet questionnaires nor microbial flora were repeated.

\section{Diet questionnaire} fructose content. Based on available literature and internet sites ${ }^{21,22}$ total fructose was calculated and an average daily dose calculated. Measured hydrogen or fecal bacteria were related to intakes $<20 \mathrm{~g} / \mathrm{d}$ or $\geq 20 \mathrm{~g} / \mathrm{d}$.

\section{Breath hydrogen and symptoms}

Breath hydrogen was measured with a validated hand held electrochemical hydrogen sensor (Bedfont Scientific, Medford NJ. USA). ${ }^{23,24}$ After baseline measurements, readings were taken every $30 \mathrm{~min}$ for $4.5 \mathrm{hrs}$. Positive tests were defined by sustained consecutive rise $\geq 10$ parts per million (ppm) above the baseline. The sum of hydrogen exhaled at each time interval after correcting for baseline was obtained for each participant.

Symptoms were also recorded at each time interval and the sum of each $30 \mathrm{~min}$ was recorded as total symptom score. The 3 cardinal symptoms of gas, bloating and cramps were qualitatively graded on a 4 point Likert scale; $0=$ none, $1=$ mild, 2 moderate and $3=$ severe. Diarrhea was recorded as 0 or 1 .

\section{Bacterial analysis}

Samples were shipped on ice to the microbiology laboratory (Macdonald site, McGill University) within $2 \mathrm{hrs}$. and were analyzed the same day. The culture methods were previously described. ${ }^{25}$ For quantification of total bifdobacteria, total lactobacilli, total anaerobes (bifidobacteria, lactobacilli, bacteroides and clostridia) and total enterobactericiae, after correcting (100 g), were introduced into an anaer-
Items were evaluated (by SG and MM) for obic jar containing gas mixture (BBL). A $1.0 \mathrm{~g}$ of feces was removed and homogenized in $4 \mathrm{~mL}$ of prepared brain heart infusion broth supplemented with yeast extract and 5-cysteine hydrochloride. A serial dilution was made and plated on Bifidobacterium (Beerens), Lactobacillus (Rogosa) agar, and Enterobacteriaceae (MacConkey). Counts (colony forming units, cfu) of total culturable anaerobes were enumerated on Reinforced Clostridial Medium agar (Fisher Scientific). Plates were incubated anaerobically for 2-3 days at $37^{\circ} \mathrm{C}$ with the exception of MacConkey (aerobically) overnight at $37^{\circ} \mathrm{C}$. All bacterial counts are reported as cfu per $\mathrm{g}$ of stool. Our primary changes of interest were focused on bifidobacteria and lactobacilli because these are the bacteria expected to increase with prebiotics, but we analyzed all four for comparison purposes.

\section{Statistical analysis}

Demographics are listed with mean \pm SD. Paired and unpaired student's t-test were used to compare the results of TBH2, TSS and Bacterial results within groups from test 2 and test 1 and test 3 and test 1 and between groups for test 2. Pearson correlations were used to assess relations between daily fructose intake breath hydrogen or lactic acid bacterial populations. Statistical analyses were carried out using statistical web page available at http://statpages.org. Statistical significance with 2 tailed tests was accepted at $\mathrm{P}<0.05$ and marginal significance at $\mathrm{P}<1>0.05$. Although, no formal power calculation was done and adaptation was previously demonstrated with 12 to 20 lactulose or lactose maldigesting subjects, respectively. ${ }^{13,14}$

\section{Results}

Demographic features of 45 volunteers are listed in Table 1. Figure 1a outlines study flow. Of the entire group, 30 (67\%) were intolerant.

Thirty-eight subjects provided stool samples (Figure 1b). Of the group F, 10 returned for test 3 (group Stop F), but did not supply stool samples.

In test 1 , there was no correlation between sum of total breath hydrogen and total symptom score in the intolerant subjects $(\mathrm{P}=1)$.

Comparison of daily fructose based on high ( $\geq 20 \mathrm{~g} / \mathrm{d}, \mathrm{N} 5$ ) or low ( $<20 \mathrm{~g} / \mathrm{d}, \mathrm{N} 21$ ) intake with total breath hydrogen showed a marginal inverse correlation in the high intake $(\mathrm{r}=-0.71$, $\mathrm{P}=0.088)$ but not in the low intake volunteers $(\mathrm{r}=-0.08, \mathrm{P}=0.6)$.

There was no correlation between total breath hydrogen and lactobacilli or bifidobacteria within the high or low intake intolerant subjects. Evaluations of bacterial findings in the entire group (N38) showed no correlations of 


\section{A. PARTICIPANTS}
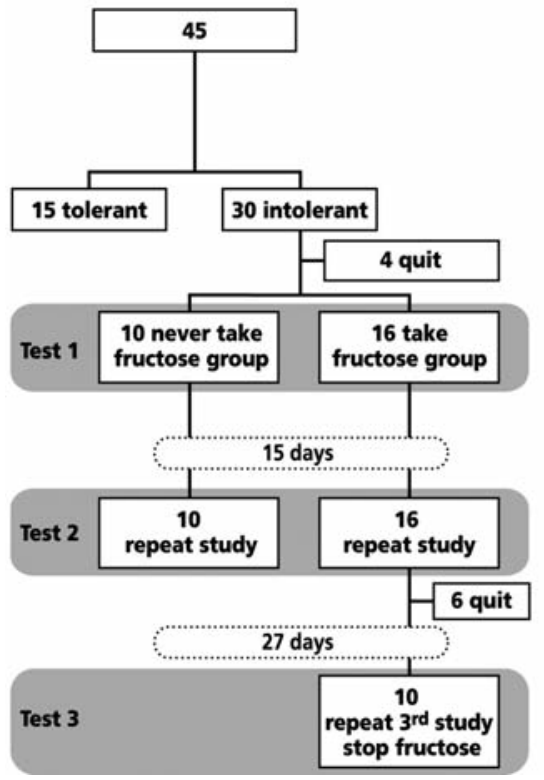

\section{B. AVAILABLE BACTERIAL ANALYSIS}

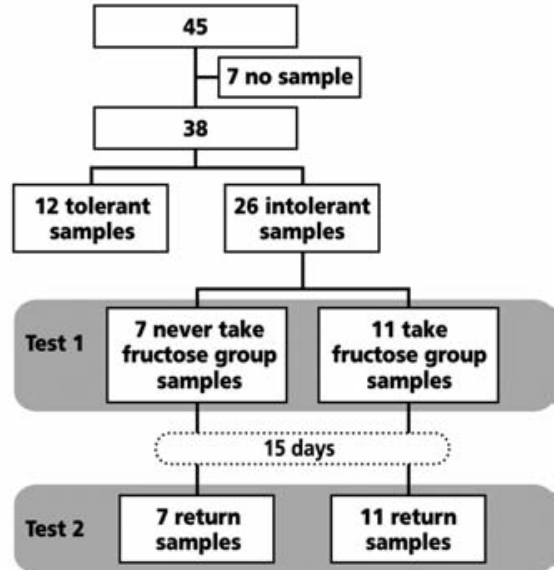

Figure 1. Outline of Volunteers and Available Stool Samples. Panel A describes the fate of 45 participants. Panel $B$ describes the number of available samples for fecal bacterial analysis. In each instance a participant quit the study because of personal reasons.

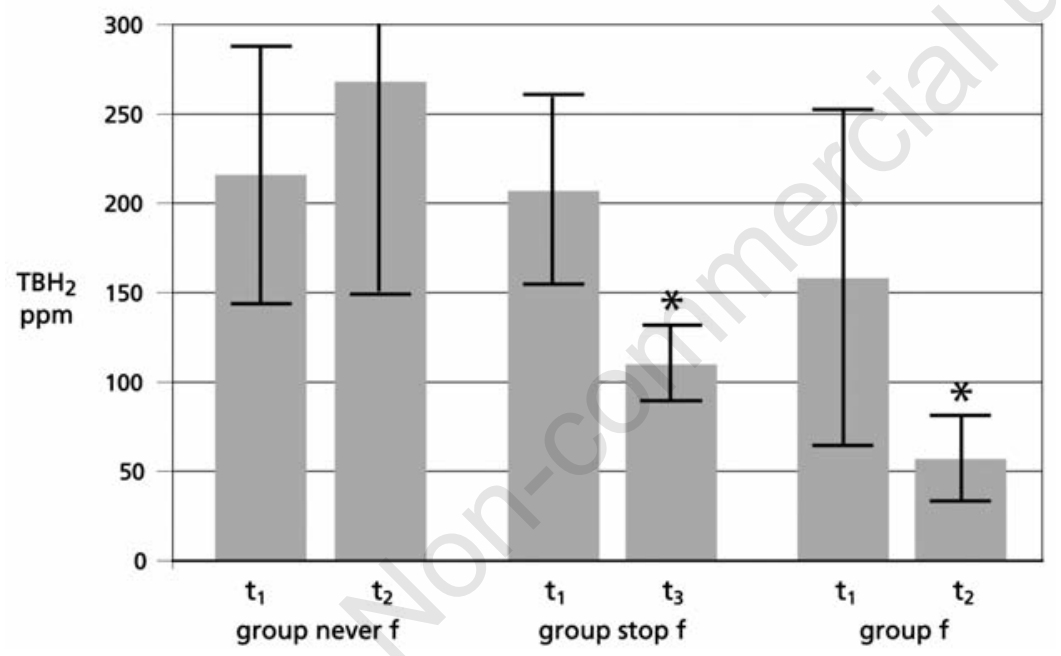

Figure 2. Comparison of Breath Hydrogen Within Groups. Total breath hydrogen (TBH2 parts per million; ppm) results are compared for 3 groups between test 1 (t1) with test 2 (t2) or test 3 (t3). Groups Never Fructose (N10), Stop Fructose (N10) and Fructose (N15, outlier excluded, see text) are displayed. Time between test 1 and test 2 was a median of 15 days and between test 3 and test 1 was a median of 42 days. The symbol * denotes statistical significance at $\mathbf{P} \leq \mathbf{0 . 0 4}$.

fructose intake with total anaerobes or enterobactericiae. There was a marginally significant negative correlation with either lactobacilli $(\mathrm{r}=-$ $0.29, \mathrm{P}=0.067)$ or bifidobacteria $(\mathrm{r}=-0.27, \mathrm{P}=$ $0.08)$.

In test 2,10 were included in group Never $\mathrm{F}$ and 16 were in group F. Total Symptom Scores for group Never F were $11.5 \pm 9.5$ (test 1 ) and $10.5 \pm 10.5$ (test 2 ) (NS).

Similar results were achieved in group $\mathrm{F}$,
$13.3 \pm 11.7$ (test 1) and $11.4 \pm 11.5$ (test 2) (NS). A sub-analysis of diarrhea scores between tests was not significant. There was no correlation between total breath hydrogen and total symptom score in either group (Figure 2).

Comparisons were made on total breath hydrogen between test 2 and test 1 . There were no significant differences in the paired analyses in the group Never F $(268 \pm 239$ vs $216 \pm 144$ ppm respectively, $\mathrm{P}=0.48)$. Within group $\mathrm{F}$, there was a non-significant numerical reduction of total breath hydrogen in test $2(96.8 \pm 165$ vs $161 \pm 182$ ppm respectively, $\mathrm{P}=0.27$ ).

Analyses of bacteria revealed no statistically significant differences between tolerant or intolerant participants, in test 2 between group Never $\mathrm{F}$ and group $\mathrm{F}$ and within groups between test 2 and test 1 (Table 2).

In test 3 , comparison of total symptom score of group Stop F, between test 3 and test 1 showed no statistical difference, (mean $10.5 \pm 12.1$ and $15.2 \pm 12.6, \mathrm{P}=0.149$ ). However, comparison of total breath hydrogen showed a significant reduction in test $3,(110 \pm 106 \mathrm{vs}$ $207 \pm 207 \mathrm{ppm}$ respectively, $\mathrm{P}=0.04)$. Since there was no rational explanation for this statistically significant reduction, we re-evaluated group $\mathrm{F}$ and found that one of the volunteers (who did not enter group Stop F) had a very high increase in hydrogen response in test 2 . When this outlier was excluded, the results of the comparison of total breath hydrogen between test 2 and 1 became significant $(57.2 \pm 47.8$ vs $158 \pm 188 \mathrm{ppm}$, $\mathrm{P}=0.036$ ). Also, comparison of total breath hydrogen at test 2 (after excluding the outlier) between group F and group Never F was highly significant $(\mathrm{P}=0.003)$, suggesting this outlier skewed the results of the study.

\section{Discussion}

This pilot study showed that symptomatic adaptation to fructose, even at doses expected to reach the colon and potentially modify colonic flora, did not take place. Quantitative bacterial changes in bifidobacteria and lactobacilli were not observed. Unexpectedly, however, breath hydrogen changes may have accompanied short term high dose fructose supplementation. The observed outcome, then for fructose supplementation is very different from that seen with lactose.

Clinical adaptation should mean improved symptoms which allows continued consumption of the initially offending sugar. Speculatively continued sugar consumption is related to some benefit to the host. This paradigm is postulated for lactose. As well, the adaptation model to lactose can be used to compare adaptive processes with other sugars like fructose, though there is no convincing evidence for this notion, as stated into the introduction. However, in the initial study the fructose dose was perhaps too low and the monosaccharide amount reaching the colon was not enough to induce bacterial effects.

In the adaptation model three putative mechanisms may modify symptoms. The first is subjective, or placebo effect of repetitive studies such that has been described for lactose. ${ }^{26}$ Improvement can be expected considering prior experience on repeat carbohydrate challenge. Herein symptoms did not improve, so a classical 
Table 2. Comparison of bacterial culture results for different groups.

Tolerant (N11)

Intolerant (N27)

\begin{tabular}{cccccccc|}
\hline Total anaerobes Enterobactericiae Bifidobacteria & Lactobacilli & Total anaerobes & Enterobactericiae & Bifidobacteria Lactobacilli \\
$8.9 \pm 3.2$ & $7.1 \pm 2.3$ & $7.5 \pm 3.6$ & $8.4 \pm 3.2$ & $9.5 \pm 2.4$ & $7.7 \pm 1.8$ & $7.9 \pm 2.7$ & $8.3 \pm 2.6$ \\
\hline
\end{tabular}

B

Group Never F (N8)

Group F (N12)

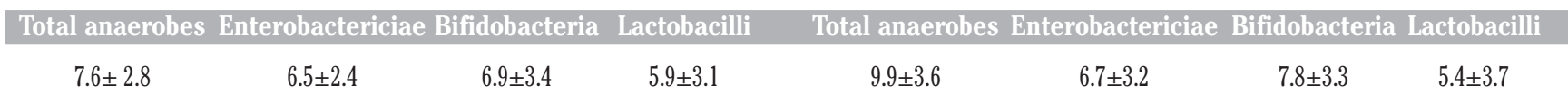

C

Group Never F (N7)*

।

Group F (N11) *

\begin{tabular}{ccccc} 
& Bifidobacteria & Lactobacilli & Bifidobacteria & Lactobacilli \\
Test 1 & $7.4 \pm 3$ & $7.6 \pm 3.1$ & $7.8 \pm 3.5$ & $9 \pm 3.2$ \\
Test 2 & $5.9 \pm 3.5$ & $5.7 \pm 3.2$ & $7.5 \pm 3.3$ & $8.1 \pm 3.7$ \\
\hline
\end{tabular}

Mean and \pm SD of cfu in $\log 10$ are shown for 4 groups of bacteria. Total anaerobes, Enterobactericiae, Bifidobacteria and Lactobacilli. Panel A compares fecal bacteria between fructose tolerant and intolerant participants in test 1; Panel B compares results in test 2 between control group Never F (no fructose given) and group F ( $30 \mathrm{~g}$ fructose twice a day for 2 weeks). Panel C shows comparison within groups Never F or F, Bifidobacteria and Lactobacilli between test 2 and test 1 . None of the differences achieved statistical significance.

placebo effect is less likely at play. The second method of adaptation is through selective metabolism and/or expansion of specific colonic bacterial populations in response to the carbohydrate. The predominance of bifidobacterial metabolism of prebiotics derived from either polymers of fructose (inulin, oligofructose or short chain fructo-oligosaccharides) or lactose (lactulose, galacto-oligosaccharides), is thought to depend on competitive advantage of this species over other bacteria. ${ }^{27}$ Promotion of bifidobacteria is considered as beneficial to the host. $^{6}$

In the case of lactose maldigesters, adaptation is thought to occur by prebiotic effects and studies show bifidobacteria and lactobacilli increase after lactose in vitro and in vivo. ${ }^{13,28-31}$ Symptomatic improvement is noted to be correlated with reduction in hydrogen production. ${ }^{32}$ In the current study no correlation of symptoms with hydrogen change was noted nor were alterations in bifidobacteria or lactobacilli.

The third possible mechanism of adaptation is via improved intestinal absorption. Improved digestion of lactose should also result in reduced hydrogen production and less symptoms. ${ }^{32}$ However, previous studies established that intestinal lactase in man is not inducible. ${ }^{15}$

It is therefore problematic that the unexpected statistically significant reductions in summed breath hydrogen in tests 3 and then 2 in response to fructose supplementation were not accompanied by parallel reduction in symptoms. Several possible mechanisms for this observation could be postulated. While a placebo (nocebo) effect cannot be eliminated other explanations could be considered. Utilization of hydrogen for manufacture of methane or hydrogen sulfide might contribute to persistent symptoms. $^{33,34}$ However, differential metabolism based on degree of fructose polymerization by lactic acid bacteria ${ }^{27}$ would make it less likely these other bacteria would significantly benefit. Instead, we favor the notion that the entry of some fructose, albeit at reduced quantity, into the colon still produced symptoms. Severity of symptoms may be minimally affected by amounts ingested. ${ }^{35}$

Regarding reduced summed breath hydrogen in the third fructose challenge test, the role played by chance cannot be ruled out. Based on previous experience with repeat challenges using lactose, however, significant reduction without intervention is unusual. ${ }^{36}$ Therefore, while statistically challenging, excluding an outlier possibly unmasked a significant effect in test 2 . As such, we speculate that perhaps regular high fructose intake may have induced intestinal carriers.

Normally fructose is transported across the upper intestinal brush border by facilitated transport through GLUT5 carrier..$^{37}$ Upon exposure to high intakes of fructose, both GLUT $5^{38}$ and GLUT2 carriers increase transport. The latter is located at the basolateral membrane and is translocated through intracellular signaling to the brush border, where its high capacity participates in enhanced transport. ${ }^{39}$ These details have been elucidated in small animal models, however, we think the current study is the first suggesting that these processes may also occur in man.

A number of weaknesses need to be addressed. First, the largely insignificant findings may be the result of under powering of the study. However, it should be noted that previous studies on lactose adaptation required at least 10 subjects to show significance. ${ }^{36}$ As well, marginal inverse bacterial and hydrogen correlations with fructose intake support a carrier hypothesis. A prebiotic effect would have positive correlation with fructose intake. Second, the implication that carriers are affected is indirect and rests on altered breath hydrogen without bacterial changes. Although bacterial counts may be affected by handling of samples, ${ }^{40}$ all were treated similarly without knowledge of fructose status. Therefore, changes should still have relevance. This study also doesn't explain why tolerance exists in some participants; for example, do their carriers have an increased capacity to transport fructose?

In conclusion, regular high fructose consumption in the short-term does not lead to symptomatic adaptation and as such it does not follow the model associated with lactose. Continued ingestion of some fructose containing foods may therefore be expected to aggravate symptoms in patients with irritable bowel syndrome. Despite failure to observe fecal bacterial changes, summed breath hydrogen diminished on rechallenges raising the speculation that fructose intestinal carriers may have been induced. In view of putative ill effects associated with fructose ingestion, clarification of enhanced absorption needs to be confirmed or refuted.

\section{References}

1. Choi YK, Johlin Jr FC, Summers RW et al. Fructose intolerance: An under-recognized problem. Am J Gastroenterol 2003;98: 134853.

2. Rumessen JJ, Gudmand-Hoyer E. Functional bowel disease: malabsorption and abdominal distress after ingestion of fructose, sorbitol and fructose-sorbitol mix- 
tures. Gastroenterology 1988;95:694-700.

3. Mishkin D, Sablouskas L, Yalovsky M, Mishkin S. Fructose and sorbitol malabsorption in ambulatory patients with functional dyspepsia. Dig Dis Sci 1997;42:25918.

4. Bray GA, Nielsen SJ, Popkin BM. Consumption of high-fructose corn syrup in beverages may play a role in the epidemic of obesity. Am J Clin Nutr 2004;79:537-43.

5. Aeberli I, Zimmermann MB, Molinari L et al. Fructose intake is a predictor of LDL particle size in overweight schoolchildren. Am J Clin Nutr 2007;86:1174-8.

6. Taylor EN, Curhan GC. Fructose consumption and the risk of kidney stones. Kidney Int 2008;73:207-12.

7. Choi HK, Curhan G. Soft drinks, fructose consumption, and the risk of gout in men: prospective cohort study. BMJ 2008;336: 309-12.

8. Kolida S, Gibson GR. Prebiotic capacity of inulin-type fructans. J Nutr 2007;137: 2503S-6.

9. Macfarlane S, Macfarlane GT. Regulation of short-chain fatty acid production. Proc Nutr Soc 2003;62:67-72.

10. Gibson GR, Probert HM, Van Loo J et al. Dietary modulation of the human colonic microbiota: updating the concept of prebiotics. Nutr Res Rev 2004;17:259-75.

11. Boateng J, Verghese M, Shackelford L et al. Selected fruits reduce azoxymethane (AOM)-induced aberrant crypt foci (ACF) in Fisher 344 male rats. Food Chem Toxicol 2007;45:725-32.

12. Lunet N, Valbuena $\mathrm{C}$, Vieira AL et al. Fruit and vegetable consumption and gastric cancer by location and histologic type: casecontrol and meta-analysis. Eur J Cancer Prev 2007;16:312-27.

13. Hertzler SR, Savaiano DA. Colonic adaptation to daily lactose feeding in lactose maldigesters reduces lactose intolerance. Am J Clin Nutr 1996;64:232-6.

14. Flourie B, Briet F, Florent $\mathrm{C}$ et al. Can diarrhea induced by lactulose be reduced by prolonged ingestion of lactulose. Am J Clin Nutr 1993:58:369-75.

15. Gilat T, Russo S, Gelman-Malacji E, Aldor TA. Lactase in man: a non adaptable enzyme. Gastroenterology 1972;62:1125-7.

16. Szilagyi A. Review article: lactose a potential prebiotic. Aliment Pharmacol Ther
2002;16:1591-602.

17. Szilagyi A, Malolepszy P, Yesovitch S et al. Fructose malabsorption may be gender dependent and fails to show compensation by colonic adaptation. Dig Dis Sci 2007;52: 2999-3004.

18. Szilagyi A, Malolepszy P, Yesovitch S et al. Inverse dose effect of pretest dietary lactose intake on breath hydrogen results and symptoms in lactase non persistent subjects. Dig Dis Sci 2005;50:2178-82.

19. Rao SSC, Attaluri A, Anderson L, Stumbo P. The ability of the normal human small intestine to absorb fructose: Evaluation by breath testing. Clin Gastroenterol Hepatol 2007;5:959-63. PMID:17625977.

20. Cooper GS, Busby MG, Fairchild AP. Measurement of lactose consumption reliability and comparison of two methods. Ann Epidemiol 1995;5:473-77.

21. Canadian Nutrient File, 2007. http//www. hc-sc.gc.ca/fn-an/nutrition/fiche-nutridata/index_e.html

22. USDA National Nutrient Database for Standard Reference, Release 19. http:// www.ars.usda.gov/main/site_main.htm?mo decode $=12-35-45-00$

23. Metz G, Gassull MA, Leeds AR et al. A simple method of measuring breathhydrogen in carbohydrate malabsorption by end-expiratory sampling. Clin Sci Mol Med 1976;50: 237-40.

24. Van der Klei-van Moorsel JM, Douwes AC, van Oeveren JP. New principle for estimation of hydrogen in expired air. Eur $\mathrm{J}$ Pediatr 1984;141:221-4

25. Jung SJ, Houde R, Baurhoo B et al. Effects of galactooligosaccharides and abifidobacteria lactis-based probiotic strain on the growth performance and fecal microflora of broiler chickens. Poultry Sci 2007;87: 169499.

26. Briet F, Pochart P, Marteau P et al. Improved clinical tolerance to chronic lactose ingestion in subjects with lactose intolerance: A placebo effect? Gut 1997;41:632-5.

27. Falony G, Calmeyn T, Leroy F, De Vuyst L. Coculture fermentation of Bifidobacterium species and Bacteroides thetaiotaomicron reveal a mechanistic insight into the prebiotic effect of inulin-type fructans. Appl Environ Microbiol 2009; 75:2312-9.

28. Jiang T, Savaiano DA. In vitro lactose fermentation by human colonic bacteria is modified by Lactobacillus acidophilus supplementation. J Nutr 1997 ;127:1489-95.

29. Jiang T, Savaiano DA. Modification of colonic fermentation by bifidobacteria and $\mathrm{pH}$ in vitro. Impact on lactose metabolism, short-chain fatty acid, and lactate production. Dig Dis Sci 1997;42:2370-7.

30. Mäkivuokko HA, Saarinen MT, Ouwehand AC, Rautonen NE. Effects of lactose on colon microbial community structure and function in a four-stage semicontinuous culture system. Biosci Biotechno Biochem 2006;70:2056-63.

31. Ito M, Kimura M. Influence of lactose on faecal microflora in lactose maldigesters. Microb Ecol Health Dis 1993;6:73-6.

32. Ladas S. Papanikos J, Arapakis G. Lactose malabsorption in Greek adults: correlation of small bowel transit time with the severity of lactose intolerance. Gut 1982;23:96873.

33. Strocchi A, Furne J, Ellis C, Levitt MD. Methanogens outcompete sulphate reducing bacteria for $\mathrm{H} 2$ in the human colon. Gut 1994;35:1098-101. PMID: 7926913

34. Levitt MD, Furne JK, Kuskowski M, Ruddy J. Stability of human methanogenic flora over 35 years and a review of insights obtained from breath methane measurements. Clin Gastroenterol Hepatol 2006;4:123-9.

35. Beyer PL, Caviar EM, McCallum RW. Fructose intake at current levels in the United States may cause gastrointestinal distress in normal adults. J Am Diet Assoc 2005;105:1559-66.

36. Szilagyi A, Rivard J, Fokeeff, K. Improved parameters of lactose maldigestion using lactulose. Dig Dis Sci 2001;46:1509-19.

37. Drozdowski LA, Thomson ABR. Intestinal sugar transport. World J Gastroenterol 2006;12:1657-70.

38. Gouyon F, Onesto C, Dalet V et al. Fructose modulates GLUT5 mRNA stability in differentiated Caco-2 cells : role of cAMP-signaling pathway and PABP (poladenylated-binding protein)-interacting protein (Paip) 2. Biochem J 2003;375:167-74.

39. Leturque A, Brot-Laroche E, Le Gall M et al. The role of GLUT2 in dietary sugar handling. J Physiol Biochem 2005;61:529-37.

40. Ott SJ, Musfeldt M, Timmis KN et al. In vitro alterations of intestinal bacterial microbiota in fecal samples during storage. Diagn Microbiol Infect Dis 2004;50:237-45. 